\title{
NOTES
}

\section{Viscosity Behavior and Conformation of Amylose in Various Solvents}

\author{
Takashi NORISUYE \\ Department of Macromolecular Science, Osaka University, \\ Toyonaka, Osaka 560, Japan
}

(Received June 22, 1994)

\begin{abstract}
KEY WORDS Amylose / Polysaccharide / Conformation / Helical Wormlike Chain / Intrinsic Viscosity / Excluded-Volume Effect /
\end{abstract}

The conformation of amylose in solution is still a subject in polysaccharide physical chemistry despite much experimental work so far done. In our recent work, ${ }^{1}$ it was found that the radius of gyration $\left\langle S^{2}\right\rangle^{1 / 2}$ and intrinsic viscosity $[\eta]$ of the polysaccharide in dimethyl sulfoxide (DMSO) vary with molecular weight $M$ in a manner predictable for a flexible chain with large excluded volume when $M$ is higher than $10^{5}$, whereas for $M$ below $10^{4}$, $[\eta]$ has an unusually weak dependence on $M$, as early observed by Burchard. ${ }^{2}$ We explained this weak $M$-dependence of $[\eta]$ as due to some helical nature of the flexible amylose chain and strong solvation ${ }^{3,4}$ of DMSO molecules onto the chain, on the basis of the theory of Yoshizaki et al. ${ }^{5}$ for $[\eta]$ of an unperturbed helical wormlike (HW) chain. ${ }^{6}$ More recently, ${ }^{7}$ the estimated HW model parameters in DMSO were shown to be consistent with the irregular helical conformation predicted from conformation analysis. ${ }^{7-9}$ Thus the locally helical chain with high flexibility in DMSO, deduced on the basis of the HW model, appears to be a fairly satisfactory molecular picture for amylose in solution. The question that remains is whether such a picture is relevant to other actual solvent systems.

Burchard $^{2}$ early observed that $[\eta]$ of amylose depends strongly on the kind of solvent even at very low $M$ where excluded- volume effects may be considered negligible. For example, $[\eta]$ of maltose (the dimer) in water is less than half that in DMSO (see also ref 1). The difference seems too large to be interpreted by invoking some difference in chain conformation. In fact, experimental evidence is available showing that the characteristic ratios at infinite $M$ in water and in DMSO are approximately the same. ${ }^{1,10} \mathrm{We}$ rather suspect that the seemingly anomalous variance $^{2}$ in $[\eta]$ with solvent at low $M$ arises primarily from different degrees of solvation. At high $M$, excluded-volume effects must be significant.

In this paper, it is shown that when these two effects are considered, the HW parameter values determining the backbone conformation of amylose in DMSO explain almost quantitatively Burchard's $[\eta]$ data $^{2}$ covering a very broad range of $M$ from 342 to $1.7 \times 10^{6}$ in a variety of solvents: water, a DMSO-acetone mixture ( $43.5 \mathrm{vol} \%$ acetone), formamide, and $0.5 \mathrm{~N}$ aqueous sodium hydroxide $(\mathrm{NaOH})$. Effects of solvation on $[\eta]$ are introduced into the theory of Yoshizaki et al. ${ }^{5}$ for unperturbed HW chains in the manner described previously ${ }^{1}$ while excluded volume effects are taken into account according to the Yamakawa-Stockmayer-Shimada scheme. ${ }^{11-13}$ In the following, the value of $[\eta]$ in the unperturbed state is denoted by $[\eta]_{0}$. 


\section{ANALYSIS}

\section{Basic Relations}

The theory of Yoshizaki et al. ${ }^{5}$ for $[\eta]_{0}$ of an HW touched-bead chain contains five parameters: the contour length $L$ of the chain (or the number $N$ of beads in the chain), the stiffness parameter $\lambda^{-1}$, the diameter $d$ of each bead, and the differential geometrical curvature $\kappa_{0}$ and torsion $\tau_{0}$ of the characteristic helix taken at the minimum energy of the HW chain. The first parameter $(=N d)$ is related to $M$ by

$$
L=M / M_{L}
$$

with $M_{\mathrm{L}}$ the molar mass per unit contour length of the chain. If solvation takes place over the entire amylose chain surface including both chain ends, then the solvated chain (hydrodynamically equivalent to an HW touchedbead chain) has an effective diameter $d^{\prime}$ and an effective contour length $L^{\prime}$ expressed by ${ }^{1}$

$$
\begin{gathered}
d^{\prime}=d+\delta \\
L^{\prime}=L+\delta=\left(M / M_{\mathrm{L}}\right)+\delta
\end{gathered}
$$

The increases of diameter and contour length due to solvation, denoted by $\delta$, have been assumed to be the same. The intrinsic viscosity of the solvated amylose chain with a given $M$ is determined by six parameters, $M_{\mathrm{L}}, \lambda^{-1}, \kappa_{0}$, $\tau_{0}, d\left(\right.$ or $\left.d^{\prime}\right)$, and $\delta$.

When the chain is perturbed by excludedvolume effect, its $[\eta]$ is generally expressed as

$$
[\eta]=\alpha_{\eta}^{3}[\eta]_{0}
$$

The viscosity expansion factor $\alpha_{\eta}$ is, in a very good approximation, ${ }^{13,14}$ a function only of the scaled excluded-volume parameter $\tilde{z}$ defined by ${ }^{11,12}$

$$
\tilde{z}=(3 / 4) K(\lambda L) z
$$

with

$$
z=(3 / 2 \pi)^{3 / 2} \lambda B(\lambda L)^{1 / 2}
$$

Here, $K(\lambda L)$ is a known function of $\lambda L$ and $B$ is the excluded-volume strength for the interaction between a pair of beads (see ref 13 or 14 for the expression of $K(\lambda L)$ and the defining equation of $B$ ). In the coil limit, $\tilde{z}$ becomes equal to the excluded-volume parameter in the two-parameter theory. ${ }^{15}$ For the relation between $\alpha_{\eta}^{3}$ and $\tilde{z}$ we use the Barrett function $^{13,16}$

$$
\alpha_{\eta}^{3}=\left(1+3.8 \tilde{z}+1.9 \tilde{z}^{2}\right)^{0.3}
$$

which has recently been found to describe $\alpha_{\eta}^{3}$ data for typical flexible polymers rather accurately. ${ }^{14}$ As may be seen from eq $4-7$, only one additional parameter $B$ is needed to evaluate excluded-volume effects on the intrinsic viscosity.

\section{Model Parameters}

In the previous analysis ${ }^{1}$ of $[\eta]$ data mentioned in the Introduction, we took the solvated dimer to be one bead as the possible smallest length scale, and obtained $M_{\mathrm{L}}=500$ $\mathrm{nm}^{-1}, \quad d^{\prime}=1.4 \mathrm{~nm}, \quad \delta=0.7 \mathrm{~nm}, \quad \lambda^{-1}=4 \mathrm{~nm}$, $\kappa_{0} / \lambda=3.5$, and $\tau_{0} / \lambda=4$ for amylose in DMSO at $25^{\circ} \mathrm{C}$; no analysis based on the monomeric length scale was feasible owing to solvation of DMSO molecules. The parameters yield a value of $3.5 \mathrm{~nm}$ for the pitch ${ }^{6}\left[=2 \pi \tau_{0} /\left(\kappa_{0}^{2}+\right.\right.$ $\left.\left.\tau_{0}^{2}\right)\right]$ of the amylose characteristic helix (at the minimum energy of the HW chain). This pitch appears to be fairly large compared to the one expected from conformation analysis, ${ }^{8,9}$ but the difference is attributable to the relatively large length scale adopted for the analysis of [ $\eta]_{\text {data }}{ }^{1,7,17}$ (see ref 7 for the consistency of the HW parameters and conformational calculations). More important here is the small $\lambda^{-1}$ value of $4 \mathrm{~nm}$ (the estimated uncertainty is $\left.\pm 0.5 \mathrm{~nm}^{1}\right)$, which is comparable to that $(4.5-5.8 \mathrm{~nm})$ of atactic poly(methyl methacrylate). ${ }^{18}$ Thus, amylose in DMSO at $25^{\circ} \mathrm{C}$ is as flexible as this polymer and can retain the helical conformation only locally.

Substituting $d^{\prime}=1.4 \mathrm{~nm}$ and $\delta=0.7 \mathrm{~nm}$ into eq 2, we get a $d$ of $0.7 \mathrm{~nm}$ for unsolvated amylose. ${ }^{1}$ We use this $d$ and the above values for $M_{L}, \lambda^{-1}, \kappa_{0} / \lambda$, and $\tau_{0} / \lambda$ in DMSO, all 
Table I. Parameters determining the intrinsic viscisity of amylose with solvation and excluded volume in various solvents

\begin{tabular}{|c|c|c|c|c|c|c|c|}
\hline Solvent & $M_{\mathrm{L}} / \mathrm{nm}^{-1}$ & $\lambda^{-1} / \mathrm{nm}$ & $\kappa_{0} / \lambda$ & $\tau_{0} / \lambda$ & $d / \mathrm{nm}$ & $\delta / \mathrm{nm}$ & $\lambda B$ \\
\hline DMSO & 500 & 4 & 3.5 & 4 & 0.7 & 0.7 & 0.38 \\
\hline $\begin{array}{l}\text { DMSO-acetone } \\
\qquad(43.5 \text { vol } \% \text { acetone })^{\mathrm{a}}\end{array}$ & & & & & & 0.65 & 0 \\
\hline Formamide $^{\mathrm{a}}$ & & & & & & 0.48 & 0.29 \\
\hline $0.5 \mathrm{~N}$ Aqueous $\mathrm{NaOH}^{\mathrm{a}}$ & & & & & & 0.40 & 1.0 \\
\hline Water $^{\mathbf{a}}$ & & & & & & 0.34 & 0.11 \\
\hline
\end{tabular}

a The values of $M_{\mathrm{L}}, \lambda^{-1}, \kappa_{0}, \tau_{0}$, and $d$ are taken to be the same as those in DMSO.

estimated at $25^{\circ} \mathrm{C}$, for the ensuing analysis of $[\eta]$ data in other solvents at $20^{\circ} \mathrm{C}$. The difference in temperature should be insignificant since $[\eta]$ of amylose is insensitive to temperature. ${ }^{2}$ The value of $\delta$ in each solvent may be evaluated from the Einstein equation $[\eta]_{0}=5 \pi N_{A}(d+\delta)^{3} /(12 M)$ with $d=0.7 \mathrm{~nm}$ and Burchard's viscosity data ${ }^{2}$ (corrected for the solution density) for the dimer on the assumption that the solvated dimer corresponds to one bead. It is then possible to compute $[\eta]_{0}$ in each solvent as a function of $M$ using the theory of Yoshizaki et al. The remaining parameter $B$ may be estimated so as to give the closest agreement between experimental $[\eta]$ at high $M\left(>10^{5}\right)$ and the values calculated from eq $4-7$ with $[\eta]_{0}$.

Table I summarizes the values of $\delta$ and $\lambda B$ in the DMSO-acetone mixture (43.5 vol\% acetone), formamide, $0.5 \mathrm{~N}$ aqueous $\mathrm{NaOH}$, and water thus estimated with the fixed values of $M_{\mathrm{L}}, \lambda^{-1}, \kappa_{0} / \lambda, \tau_{0} / \lambda$, and $d$ and also those in DMSO determined from our previous data. ${ }^{1}$ The solvent layer thickness $(=\delta / 2)$ for each solvent system seems to be of reasonable magnitude when we consider the size of one (main) solvent molecule. The $\lambda B$ value 0.38 in DMSO may be compared to the value of 0.35 that can be obtained from the previous $\left\langle S^{2}\right\rangle$ data $^{1}$ with the same HW model parameter values. Since, according to Burchard, ${ }^{2}$ the DMSO-acetone mixture is a theta solvent for amylose (DMSO is a good solvent, while acetone is a precipitant), $\lambda B$ in the mixture has

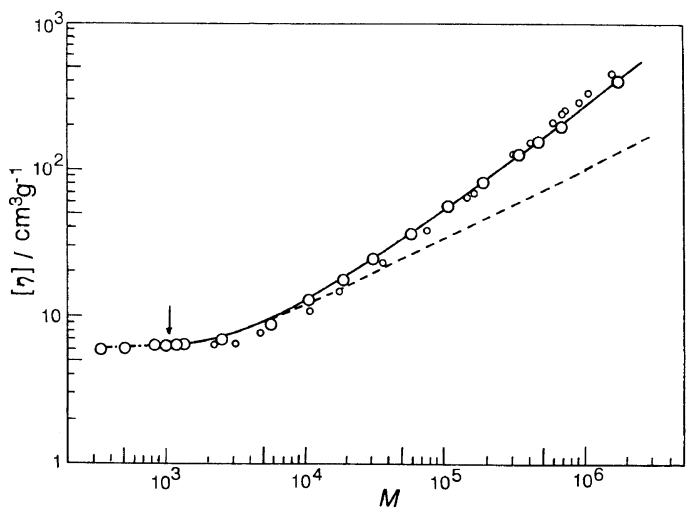

Figure 1. Comparison between experimental and theoretical $[\eta]$ values for amylose in DMSO. Larger circles, data of Nakanishi et al. ${ }^{1}$; smaller cicles, data of Burchard ${ }^{2}$; solid line, theoretical values for the perturbed HW chain with the parameter values in Table I; dashed line, theoretical values for the unperturbed HW chain. The dot-dash line connects the theoretical values for $N=1$ and 2 (the arrow).

been set equal to zero. It should be noted that in a mixed solvent system, strong solvation of one solvent component does not always bring about strong excluded-volume interaction.

\section{COMPARISON BETWEEN THEORY AND EXPERIMENT}

Figure 1 compares our previous $[\eta]$ data $^{1}$ (the larger circles) and Burchard's ${ }^{2}$ (the smaller circles) for synthetic amylose in DMSO with the theoretical values (the solid line) for the perturbed HW chain having the model parameters in Table I. The dashed line rep- 


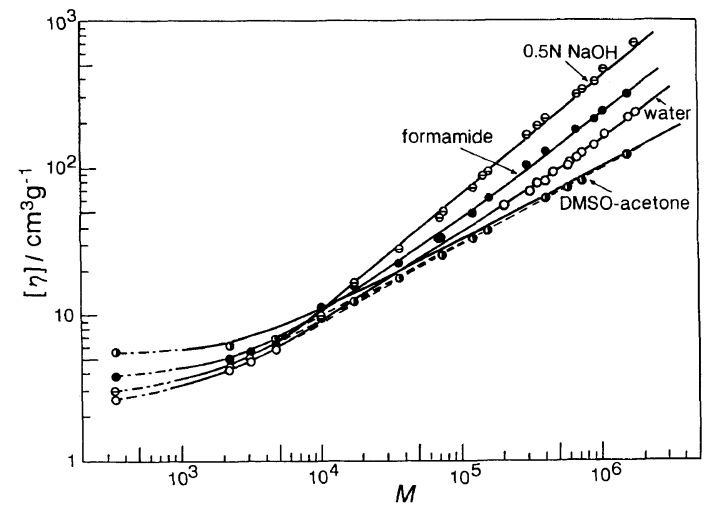

Figure 2. Comparison between experimental and theoretical $[\eta]$ values for amylose in indicated solvents. The solid curves represent theoretical HW values for the parameters in Table I, while the dashed curves (for formamide, $0.5 \mathrm{~N}$ aqueous $\mathrm{NaOH}$, and water) refer to the unperturbed state. The dot-dash line has the same meaning as that in Figure 1. The experimental data are due to Burchard. ${ }^{2}$

resents the corresponding theoretical values in the unperturbed state $(B=0)$. For clarity, the theoretical values for $N>2$ have been connected as indicated and those for $N=1$ and 2 by a dot-dash line. The arrow points the $M$ value corresponding to $N=2$. It can be seen that the solid curve closely fits the larger circles over the entire range of $M$ studied (see ref 1 for the qualitative explanation of the very weak $M$-dependence of $[\eta]$ in the oligomer region). Excluded-volume effects on $[\eta]$ are seen to become significant when $M$ exceeds about $10^{4}$.

In Figure 2, Burchard's data in the DMSO-acetone mixture of $43.5 \mathrm{vol} \%$ acetone, formamide, $0.5 \mathrm{~N}$ aqueous $\mathrm{NaOH}$, and water are compared with the calculated values; data in water for $M$ between $10^{4}$ and $1.6 \times 10^{5}$ are unavailable. The solid lines again represent the theoretical [ $\eta]$ values for the parameter sets in Table I, while the dashed lines for att the solvents (other than the DMSO-acetone mixture) refer to the unperturbed state (the dot-dash lines have the same meaning as in Figure 1). Except for the mixed theta solvent, the agreement between theory and experiment is quite satisfactory. Thus, we may conclude that the same backbone conformation as that in DMSO accounts for the viscosity behavior of amylose at least in the three solvents (formamide, $0.5 \mathrm{~N}$ aqueous $\mathrm{NaOH}$, and water) over the entire range of molecular weight examined. The unperturbed lines for these three solvents (i.e., the dashed ones), which significantly differ from one another in the oligomer region owing to solvation effects, become indistinguishable above $M \sim 10^{5}$.

The data points for the DMSO-acetone mixture appear considerably below the corresponding theoretical curve in the range of $M$ between $4 \times 10^{3}$ and $5 \times 10^{4}$. Although this curve around $M=10^{4}$ can be lowered by choosing a smaller value for $\lambda^{-1}$ or $\tau_{0} / \lambda$, such a change in parameter gives rise to a pronounced downward deviation of the theoretical line at high $M$. Since the DMSOacetone data for $M>3 \times 10^{4}$ fall on the unperturbed line for water, it is likely that our model for solvation is too simple to explain precisely the $[\eta]$ data around $M=10^{4}$ in the mixed theta solvent. However, the discrepancy between these data and the theoretical solid curve in the range of $M$ from $4 \times 10^{3}$ to $5 \times 10^{4}$ is almost parallel with what may be seen in Figure 1 for Burchard's DMSO data in the same $M$ range.

The HW model parameters in Table I yield $8.3 \times 10^{-4} \mathrm{~nm}^{2}$ for the unperturbed dimensions $\left(\left\langle S^{2}\right\rangle_{0} / M\right)_{\infty}$ in all the solvents examined, when use is made of the expression ${ }^{6}$

$\left(\left\langle S^{2}\right\rangle_{0} / M\right)_{\infty}=\left(4 \lambda^{2}+\tau_{0}^{2}\right) /\left[6 \lambda M_{L}\left(4 \lambda^{2}+\kappa_{0}^{2}+\tau_{0}^{2}\right)\right]$

for the HW model chain. Here, $\left\langle S^{2}\right\rangle_{0}$ denotes the unperturbed mean-square radius of gyration and the subscript $\infty$ attached to $\left\langle S^{2}\right\rangle_{0} / M$ signifies the limit of infinite $M$. The estimated $\left(\left\langle S^{2}\right\rangle_{0} / M\right)_{\infty}$ value agrees closely with the light scattering value ${ }^{2}$ of $8.12 \times 10^{-4} \mathrm{~nm}^{2}$ for a synthetic (nearly monodisperse) amylose sample with $M=5.29 \times 10^{5}$ in the DMSOacetone mixture, indicating that the $\mathrm{HW}$ parameters in DMSO are consistent with the 
light scattering data in the theta solvent at least for high $M$.

According to Everett and Foster ${ }^{19}$ and Banks and Greenwood, ${ }^{20,21} 0.33 \mathrm{M}$ aqueous potassium chloride $(\mathrm{KCl})$ is another theta solvent for amylose. The $[\eta]_{0}$ data of the latter group in this solvent $\left(22.5^{\circ} \mathrm{C}\right)$ for $M$ between $8.3 \times 10^{4}$ and $2.3 \times 10^{6}$ (not shown) come systematically above those in the DMSOacetone mixture or the unperturbed line for pure water in Figure 2. The difference, which is about $15 \%$ in $[\eta]_{0} / M^{1 / 2}$, implies that $\left(\left\langle S^{2}\right\rangle_{0} / M\right)_{\infty}$ in $0.33 \mathrm{M}$ aqueous $\mathrm{KCl}$ is larger by about $10 \%$ than that in the DMSO-acetone mixture (provided the Flory viscosity factor is the same). A similar difference in $\left(\left\langle S^{2}\right\rangle_{0} / M\right)_{\infty}$ might also exist between $0.33 \mathrm{M}$ aqueous $\mathrm{KCl}$ and pure water, but it does not seem important in the present context, if any. The data of Banks and Greenwood in the aqueous salt can be fitted by the theory of Yoshizaki et al. ${ }^{5}$ $(B=0)$ regardless of $\delta$ smaller than $0.5 \mathrm{~nm}$ when $\lambda^{-1}$ is changed to $4.4 \mathrm{~nm}$, a value within the range of uncertainty ${ }^{1}$ (i.e., $4 \pm 0.5 \mathrm{~nm}$ ) originally estimated.

\section{CONCLUSIONS}

The present analysis has shown that the published $[\eta]$ data for amylose in various solvents can be explained almost quantitatively over a wide range of $M$ by the theory of Yoshizaki et al. ${ }^{5}$ for HW bead chains when solvation and excluded volume effects are considered. The HW parameter values used are essentially the same for all the solvents studied (i.e., DMSO, a DMSO-acetone mixture of $43.5 \mathrm{vol} \%$ acetone, formamide, $0.5 \mathrm{~N}$ aqueous $\mathrm{NaOH}$, pure water, and $0.33 \mathrm{M}$ aqueous $\mathrm{KCl}$ ) and consistent not only with simulated $\left\langle S^{2}\right\rangle$ data $^{7}$ but also with light scattering $\left\langle S^{2}\right\rangle$ data $^{1,2}$ in DMSO and in the DMSO-acetone mixture. Thus, the locally helical, flexible chain previously deduced from the HW parameters in $\mathrm{DMSO}^{1}$ is the molecular picture common to amylose in a variety of solvents. It should be pointed out, however, that the detailed helical conformation in actual solution, viewed on the monomeric length scale, still remains to be seen by static experiment.

\section{REFERENCES}

1. Y. Nakanishi, T. Norisuye, A. Teramoto, and S. Kitamura, Macromolecules, 26, 4220 (1993).

2. W. Burchard, Makromol. Chem., 64, 110 (1963).

3. B. Casu, M. Reggiani, G. G. Gallo, and A. Vigevani, Tetrahedron Lett., No. 27, 2253 (1965).

4. B. Casu, M. Reggiani, G. G. Gallo, and A. Vigevani, Tetrahedron, 22, 3061 (1966).

5. T. Yoshizaki, I. Nitta, and H. Yamakawa, Macromolecules, 21, 165 (1988).

6. H. Yamakawa, in "Molecular Conformation and Dynamics of Macromolecules in Condensed Systems," M. Nagasawa, Ed., Elsevier Amsterdam, 1988, p 21.

7. Y. Nakata, S. Kitamura, K. Takeo, and T. Norisuye, Polym. J., 26, 1085 (1994).

8. R. C. Jordan, D. A. Brant, and A. Cesàro, Biopolymers, 17, 2617 (1978).

9. S. Kitamura, T. Okamoto, Y. Nakata, T. Hayashi, and T. Kuge, Bipolymers, 26, 537 (1987).

10. T. Norisuye, Food Hydrocoll., in press.

11. H. Yamakawa and W. H. Stockmayer, J. Chem. Phys., 57, 2843 (1972).

12. J. Shimada and H. Yamakawa, J. Chem. Phys., 85, 591 (1986).

13. F. Abe, Y. Einaga, and H. Yamakawa, Macromolecules, 26, 1891 (1993).

14. F. Abe, K. Horita, Y. Einaga, and H. Yamakawa, Macromolecules, 27, 725 (1994).

15. H. Yamakawa, "Modern Theory of Polymer Solutions," Haper \& Row, New York, N.Y., 1971.

16. A. J. Barrett, Macromolecules, 17, 1566 (1984).

17. M. Fujii, K. Nagasaka, J. Shimada, and $H$. Yamakawa, Macromolecules, 16, 1613 (1983).

18. Y. Fujii, Y. Tamai, T. Konishi, and H. Yamakawa, Macromolecules, 24, 1608 (1991).

19. W. W. Everett and J. F. Foster, J. Am. Chem. Soc., 81, 3459, 3464 (1959).

20. W. Banks and C. T. Greenwood, Makromol. Chem., 67, 49 (1963).

21. W. Banks and C. T. Greenwood, Carbohydr. Res., 7, 349 (1968). 